\title{
Modeling Claims Data with Composite Stoppa Models
}

\author{
Enrique Calderín-Ojeda ${ }^{a}$ and Chun Fung $\mathrm{Kwok}^{b}$ \\ ${ }^{a}$ Centre for Actuarial Studies, Department of Economics, University of \\ Melbourne, Australia \\ ${ }^{b}$ Department of Mathematics and Statistics, University of Melbourne, Australia
}

\begin{abstract}
In this paper, a new class of composite model is proposed for modeling actuarial claims data of mixed sizes. The model is developed using the Stoppa distribution and a mode-matching procedure. The use of the Stoppa distribution allows for more flexibility over the thickness of the tail and the mode-matching procedure gives a simple derivation of the model compositing with a variety of distributions. In particular, the Weibull-Stoppa and the Lognormal-Stoppa distributions are investigated. Their performance is compared with existing composite models in the context of the well-known Danish fire insurance dataset. The results suggest the composite Weibull-Stoppa model outperforms the existing composite models in all seven goodness-of-fit measures considered.
\end{abstract}

Key Words: Stoppa distribution; Lognormal distribution; Weibull distribution; Danish fire losses; Spliced model; Goodness-of-fit; Composite model; Bootstrap.

\section{Acknowledgements}

The authors would like to express their gratitude to an anonymous referee for the relevant and useful comments that have improved this paper. This paper was supported by The University of Melbourne ECR Grant Scheme. 


\section{Introduction}

The modeling of claims data is central to many applications in general insurance. The claims faced by insurers are often a mixture of moderate and large claims. The distribution is typically unimodal, highly positively skewed, and has a thick upper tail. In the case where the large claims are more dominant, traditionally the Pareto distribution has been considered. On the other hand, when the moderate claims are more dominant, continuous parametric families with positive support such as Gamma, Lognormal, Inverse Gaussian and Weibull have been used (Klugmann et al., 2008). Nevertheless, no standard parametric model seems to provide an acceptable fit to both small and large claims as probability distributions that provide a good overall fit can perform badly fitting a local region. In particular, the tail of the claims distribution can often be underestimated.

To overcome this issue, composite parametric models that use Lognormal (Cooray and Ananda, 2005) or Weibull (Ciumara, 2006) up to an unknown single threshold value, estimated from the data, and a two-parameter Pareto density thereafter have been considered. In both approaches, continuity and differentiability conditions were imposed at the threshold to yield a smooth density function and to reduce the number of parameters to be estimated. The resulting models are similar in shape to the Lognormal and Weibull distributions but with a thicker tail. These models give a significantly better fit to claims data compared with the standard parametric families. However, since these models use fixed and a priori known mixing weights, they can be very restrictive. In light of that, Scollnik (2007) suggested an improvement to the composite Lognormal-Pareto model by incorporating unrestricted mixing weights as coefficients in each component (see also Nadarajah and Bakar, 2014). Similar modification has been made by Scollnik and Sun (2012) to the composite Weibull-Pareto model.

In this work, a new class of composite models for modeling claims data of mixed sizes is proposed. This model is based on the Stoppa distribution. The Stoppa distribution, not sufficiently well-described in the English language literature, is a generalization of the Pareto distribution (Stoppa, 1990). It is obtained by applying a power transformation to the cumulative

distribution function (cdf) of the Pareto distribution. One main feature of the Stoppa distribution is that it presents a heavier tail than the classical Pareto distribution when the additional shape parameter is larger than one. Recently, Burkhauser et al. (2010) used it to model topcoded income values 
and yielded positive outcomes.

The construction of the proposed composite-Stoppa model uses a modematching procedure. The first component of the spliced model (e.g. Lognormal or Weibull) is used up to the modal value, which can be estimated from the data, and the adequate truncation of the Stoppa distribution is used thereafter. To avoid potential confusion, note that "mode-matching" in this paper refers to matching the modes of the two distributions of the spliced model rather than matching the model mode to the empirical mode. In general, there may be some distance between the mode fitted and the actual mode computed from the data as it will be seen later in the numerical applications section. This methodology, like the traditional continuitydifferentiability method, incorporates unrestricted mixing weights but in addition, it gives a simpler derivation of the model over the traditional method when the mode of the distribution actually has a nice form. The key idea, as compared with matching the continuity and differentiability conditions, is based on the assumption that it is easier to match the modal value since the mode of the distributions that are commonly considered in the content of actuarial modeling often has a much simpler expression, than the second derivative of the corresponding density function. This can serve as a useful alternative when the differentiability condition is hard to solve or when one wishes to conduct a pilot study. In order to concretely evaluate the composite Stoppa model, two composite families, namely the Lognormal-Stoppa and the Weibull-Stoppa families are derived and investigated. These choices are made so that the result can be compared with existing composite models.

The structure of this paper is as follows. In Section 2 a short review on composite Pareto models with unrestricted mixing weights is provided. Next in Section 3 some existing results on the Stoppa distribution are given. Later in Section 4, the genesis of two composite Stoppa models, LognormalStoppa and Weibull-Stoppa, is described. Afterwards, in Section 5 numerical illustrations are provided based upon the well-known Danish fire insurance dataset. Here the composite Stoppa model is compared with existing composite models from two points of view, theoretical plausibility and practical considerations. Finally conclusions are given in the last Section. 


\section{Composite Pareto Models with unrestricted mixing weights}

Scollnik (2007) improved the composite model given in Cooray and Ananda (2005) by incorporating unrestricted mixing weights. The density function of the composite model can be written as

$$
f(x)=\left\{\begin{array}{lr}
r f_{1}^{*}(x), & 0<x \leq \theta \\
(1-r) f_{2}^{*}(x), & \theta<x<\infty
\end{array}\right.
$$

with $0 \leq r \leq 1, f_{1}^{*}(x)=\frac{f_{1}(x)}{F_{1}(\theta)}$ and $f_{2}^{*}(x)=\frac{f_{2}(x)}{1-F_{2}(\theta)}$ are adequate truncations of the probability density functions $f_{1}$ and $f_{2}$ up to and thereafter an unknown threshold value $\theta$ where $F_{1}(\theta)$ and $F_{2}(\theta)$ denote the cumulative distribution function (cdf) of $f_{1}$ and $f_{2}$ at $\theta$ respectively. Then (1) can be seen as a convex sum of two density functions and hence it is in a form of a mixture model. After imposing the continuity condition (i.e. $f\left(\theta^{-}\right)=f\left(\theta^{+}\right)$), we have

$$
r=\frac{f_{2}(\theta) F_{1}(\theta)}{f_{2}(\theta) F_{1}(\theta)+f_{1}(\theta)\left(1-F_{2}(\theta)\right)} .
$$

Next, differentiability condition at $\theta$ was also imposed in order to make (1) smooth and to reduce the number of parameters.

\section{$2.1 \quad$ Lognormal-Pareto Models}

Let

$$
f_{1}(x)=\frac{1}{\sqrt{2 \pi} x \sigma} \exp \left(-\frac{1}{2}\left(\frac{\ln x-\mu}{\sigma}\right)^{2}\right), \quad x>0
$$

be the probability density function (pdf) of a two-parameter Lognormal distribution, where $\mu \in \mathbb{R}, \sigma>0$, and

$$
f_{2}(x)=\frac{\alpha \theta^{\alpha}}{x^{\alpha+1}}, \quad x>\theta
$$

be the pdf of a two-parameter Pareto distribution, where $\alpha, \theta>0$. 
The density function of this composite model is given by

$$
f(x)=\left\{\begin{array}{lc}
r \frac{f_{1}(x)}{\Phi(\alpha \sigma)}, & 0<x \leq \theta \\
(1-r) f_{2}(x), & \theta<x<\infty
\end{array}\right.
$$

with $0 \leq r \leq 1$ and $\Phi(\cdot)$ denotes the cdf of the standard normal distribution. By allowing for continuity and differentiability at $\theta$, we have that

$$
\begin{aligned}
r & =\frac{\sqrt{2 \pi} \alpha \sigma \Phi(\alpha \sigma) \exp \left(\frac{1}{2}(\alpha \sigma)^{2}\right)}{\sqrt{2 \pi} \alpha \sigma \Phi(\alpha \sigma) \exp \left(\frac{1}{2}(\alpha \sigma)^{2}\right)+1} \quad \text { and } \\
\alpha \sigma & =\frac{\ln \theta-\mu}{\sigma} .
\end{aligned}
$$

Then (5) is defined by means of the threshold $\theta$, a tail index $\alpha$ and a small loss parameter $\sigma$.

Scollnik (2007) also considered another composite model, the LognormalType II Pareto (Lomax) with pdf

$$
f(x)=\left\{\begin{array}{lc}
r \frac{f_{1}(x)}{\Phi(\mathcal{A})}, & 0<x \leq \theta \\
(1-r) f_{2}(x), & \theta<x<\infty
\end{array}\right.
$$

where $\mathcal{A}=\frac{\ln \theta-\mu}{\sigma}=\left(\frac{\alpha \theta-\lambda}{\lambda+\theta}\right) \sigma$, and $f_{2}(x)$ is the pdf of the Type II Pareto given by

$$
f_{2}(x)=\frac{\alpha(\lambda+\theta)^{\alpha}}{(\lambda+x)^{\alpha+1}}, \quad x>\theta
$$

where the parameters are $\theta>0, \alpha>0$, and $\lambda>-\theta$.

After imposing the continuity and differentiability requirements at $\theta$ a smooth four-parameter density is obtained, $r$ is now provided by

$$
r=\frac{\sqrt{2 \pi} \alpha \theta \sigma \Phi(\mathcal{A}) \exp \left(\frac{1}{2} \mathcal{A}^{2}\right)}{\sqrt{2 \pi} \alpha \theta \sigma \Phi(\mathcal{A}) \exp \left(\frac{1}{2} \mathcal{A}^{2}\right)+\lambda+\theta} .
$$

Note that this model nests the composite Lognormal-Pareto model if $\lambda=0$. 


\subsection{Weibull-Pareto Models}

Let

$$
f_{1}(x)=\frac{\tau}{x}\left(\frac{x}{\phi}\right)^{\tau} \exp \left(-\left(\frac{x}{\phi}\right)^{\tau}\right), \quad x>0
$$

be the pdf of a two-parameter Weibull distribution, where $\phi, \tau>0$, and

$$
f_{2}(x)=\frac{\alpha \theta^{\alpha}}{x^{\alpha+1}}, \quad x>\theta,
$$

be the pdf of a two-parameter Pareto distribution, where $\theta, \alpha>0$.

Scollnik and Sun (2012) constructed a composite model with pdf

$$
f(x)=\left\{\begin{array}{lc}
r \frac{f_{1}(x)}{F_{1}(\theta)}, & 0<x \leq \theta \\
(1-r) f_{2}(x), & \theta<x<\infty
\end{array}\right.
$$

with $0 \leq r \leq 1$ and $F_{1}(\theta)$ is the cdf of the Weibull distribution at $\theta$. By allowing for continuity and differentiability at $\theta$, a three-parameter model is obtained. Now $r$ is provided by

$$
r=\frac{\alpha \exp (\mathcal{B})-\alpha}{\alpha \exp (\mathcal{B})+\tau}, \quad \text { where } \quad \mathcal{B}=\left(\frac{\theta}{\phi}\right)^{\tau}=\frac{\alpha}{\tau}+1
$$

In a similar fashion as in the Lognormal-Pareto composite family, Scollnik and Sun (2012) also considered the composite Weibull-Type II Pareto (Lomax) with pdf

$$
f(x)= \begin{cases}r \frac{f_{1}(x)}{F_{1}(\theta)}, & 0<x \leq \theta \\ (1-r) f_{2}(x), & \theta<x<\infty\end{cases}
$$

where $f_{2}(x)$ is the pdf of the Type II Pareto and it is given by

$$
f_{2}(x)=\frac{\alpha(\lambda+\theta)^{\alpha}}{(\lambda+x)^{\alpha+1}}, \quad x>\theta, \theta>0, \alpha>0, \text { and } \lambda>-\theta .
$$

Then by imposing the continuity and differentiability conditions at $\theta$, a fourparameter smooth density function is derived. Here $r$ is provided by

$$
r=\frac{\alpha}{\tau}\left(\frac{\lambda+\theta}{\theta} \frac{\mathcal{C}}{\exp (\mathcal{C})-1}+\frac{\alpha}{\tau}\right)^{-1}
$$

where $\mathcal{C}=\left(\frac{\theta}{\phi}\right)^{\tau}=\frac{\alpha \theta-\lambda}{(\lambda+\theta) \tau}+1$, clearly, this model nests the composite Weibull-Pareto model if $\lambda=0$. 


\section{Generalizing the Pareto distribution}

Although not sufficiently well-described in the English language literature, a generalization of the Pareto distribution was proposed by Stoppa (1990). The methodology to derive this family of distributions involves applying a power transformation to the Pareto cdf. The cdf of the Stoppa distribution is given by

$$
F(x)=\left[1-\left(\frac{x}{x_{0}}\right)^{-\delta}\right]^{\gamma}, \quad 0<x_{0} \leq x,
$$

where $\delta, \gamma>0$ specify the shape of the distribution and $x_{0}$ is the minimum possible value. The classical Pareto distribution is obtained when $\gamma=1$. The pdf of the Stoppa distribution is provided by

$$
f(x)=\gamma \delta x_{0}^{\delta} x^{-(\delta+1)}\left[1-\left(\frac{x}{x_{0}}\right)^{-\delta}\right]^{\gamma-1}, \quad 0<x_{0} \leq x .
$$

Some properties of this distribution can be found in Kleiber and Kotz (2003). In this regard, the $k^{t h}$ order moment exists for $k<\delta$ is given by

$$
E\left(X^{k}\right)=\gamma x_{0}^{k} B e\left(1-\frac{k}{\delta}, \gamma\right)
$$

where $B e(\cdot, \cdot)$ represents the beta function defined by

$$
B e(a, b)=\int_{0}^{1} z^{a-1}(1-z)^{b-1} d z \quad \text { with } \quad a, b>0 .
$$

Additionally, the quantile function can be easily derived,

$$
F^{-1}(u)=x_{0}\left(1-u^{1 / \gamma}\right)^{-1 / \delta}, \quad 0<u<1 .
$$

As compared with the Pareto distribution, the Stoppa distribution is more flexible since it includes an additional shape parameter $\gamma$ that allows for unimodality for $\gamma>1$ and zeromodality when $\gamma \leq 1$. For the former case the mode is located at

$$
x_{\text {Mode }}=x_{0}\left(\frac{1+\gamma \delta}{1+\delta}\right)^{1 / \delta}, \gamma>1
$$


whereas for the latter case it is at $x_{0}$. Figure 3 shows the effect of increasing the shape parameter $\gamma$ on probability density function of the Stoppa distribution while holding $x_{0}$ and $\delta$ fixed. As $\gamma \geq 1$ increases, the mode moves to the right producing a thicker tail.

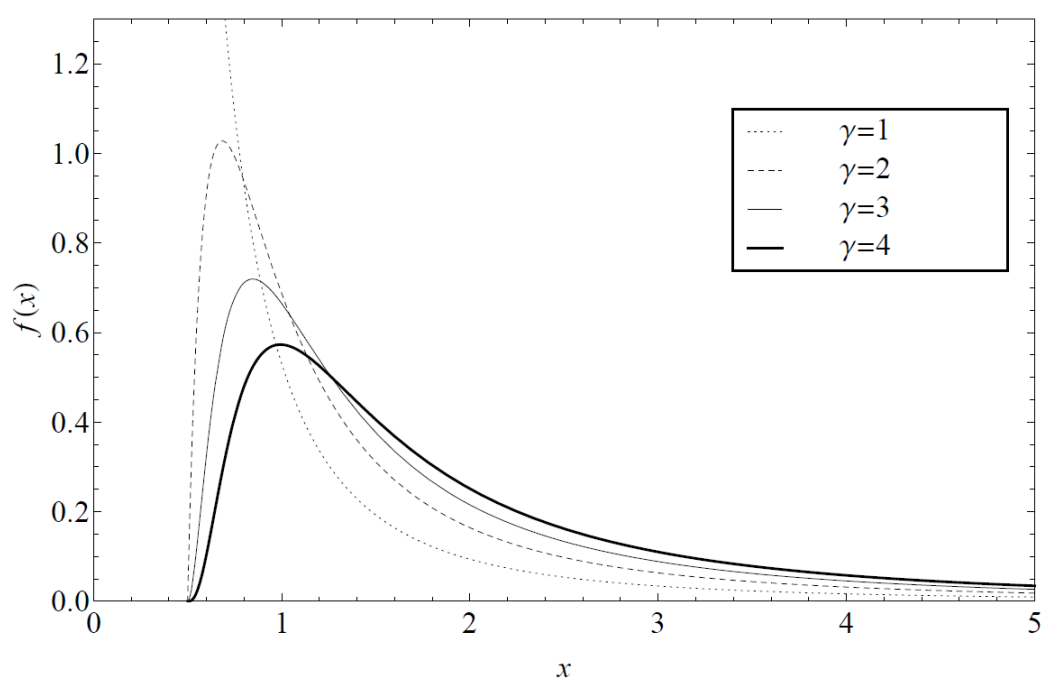

Figure 1: Stoppa densities for $x_{0}=0.5, \delta=1.5$, and $\gamma=1$ (dotted), $\gamma=2$ (dashed), $\gamma=3$ (solid thin) and $\gamma=4$ (solid thick).

\section{The Composite Stoppa Models}

The composite Pareto models described use the Pareto distribution above the threshold value. As both classical and Type II Pareto distributions are monotonically decreasing, the threshold estimate is always greater than the modal value of the composite model. This formulation is natural as it is the intention to use Pareto distribution to model the large claims.

Yet, one other viewpoint that could be useful is to consider a composite model where the splice point is at the mode of the data. Under this construction, the use of two distributions for the different sides of the claims distribution is in effect modeling how fast the probability decreases from the 
mode to either ends of the distribution. This addresses the asymmetric nature of the claims distribution resulting from the asymmetric sizes of the state space on both sides of the mode of the data. As a result, the data support of both sides of the spliced model are more balanced, compared with for example, a composite Pareto model with a large threshold estimate. This has the benefits that the fitting of the model is more robust to new data, especially data of large claims, and that the information in the moderate-tolarge claims about the large claims can be captured, through modeling the transition between the two classes of claims sizes.

Using a mode-matching procedure, the construction of the composite Stoppa model is now given. Note that this procedure incorporates unrestricted mixing weights into the model. The first component of the spliced model is used up to the modal value (which is to be estimated from the data) and the adequate truncation of the Stoppa distribution given in (7) thereafter. Then, the density function of the composite Stoppa model can be written as

$$
f(x)=\left\{\begin{array}{lr}
r f_{1}^{*}(x), & 0<x \leq x_{m} \\
(1-r) f_{2}^{*}(x), & x_{m}<x<\infty
\end{array}\right.
$$

with $0 \leq r \leq 1, f_{1}^{*}(x)=\frac{f_{1}(x)}{F_{1}\left(x_{m}\right)}$ an adequate truncation of the probability density functions $f_{1}$ up to the modal value, where $F_{1}\left(x_{m}\right)$ is the cdf of $f_{1}$ evaluated at $x_{m}$, and $f_{2}^{*}(x)=\frac{f_{2}(x)}{1-F_{2}\left(x_{m}\right)}$ a suitable truncation of the Stoppa distribution, where $1-F_{2}\left(x_{m}\right)$ is the survival function evaluated at $x_{m}$. Again, $(9)$ is in a form of a mixture model.

In place of the usual continuity and differentiability conditions, a modematching procedure is used. This procedure ensures the continuity and differentiability conditions are satisfied. In addition, it gives a simpler derivation of the model compositing with any distributions with a mode that has a closed form expression. The mode-matching conditions are given as follows.

Denote the modes of the distributions used by the first and second components of the composite model by $x_{m}^{f i r s t}, x_{m}^{\text {second }}$ respectively. Then the modematching conditions are:

$$
\begin{aligned}
x_{m}^{\text {first }} & =x_{m}^{\text {second }} \\
r f_{1}^{*}\left(x_{m}^{f i r s t}\right) & =(1-r) f_{2}^{*}\left(x_{m}^{\text {second }}\right)
\end{aligned}
$$


Clearly, (11) implies the continuity condition is satisfied, and since the equality in (10) allows us to drop the "first" and "second" labels, the simple expression for the mixing weight, as seen in the other existing composite models, is preserved and is given by

$$
r=\frac{f_{2}\left(x_{m}\right) F_{1}\left(x_{m}\right)}{f_{2}\left(x_{m}\right) F_{1}\left(x_{m}\right)+f_{1}\left(x_{m}\right)\left(1-F_{2}\left(x_{m}\right)\right)} .
$$

Next, note that for unimodal distribution, the derivative at the mode is zero, so it is clear the differentiability condition is also satisfied.

\subsection{Lognormal-Stoppa Model}

The composite Lognormal-Stoppa model will be derived in terms of the mixture model (9). Its density function is given by

$$
f(x)= \begin{cases}r \frac{\frac{1}{\sqrt{2 \pi} x \sigma} \exp \left(-\frac{1}{2}\left(\frac{\ln x-\mu}{\sigma}\right)^{2}\right)}{\Phi\left(\frac{\ln x_{m}-\mu}{\sigma}\right)}, & 0<x \leq x_{m} \\ (1-r) \frac{\gamma \delta x_{0}^{\delta} x^{-(\delta+1)}\left[1-\left(\frac{x}{x_{0}}\right)^{-\delta}\right]^{\gamma-1}}{1-\left[1-\left(\frac{x_{m}}{x_{0}}\right)^{-\delta}\right]^{\gamma}}, & x_{m}<x<\infty\end{cases}
$$

with $\mu \in \mathbb{R}, \sigma>0, \gamma>1, \delta>0,0 \leq r \leq 1$ and $\Phi(\cdot)$ denotes the cdf of the standard normal distribution.

Using the mode--matching procedure, 10 gives

$$
\sigma=\sqrt{\mu-\ln \left[x_{0}\left(\frac{1+\gamma \delta}{1+\delta}\right)^{1 / \delta}\right]}
$$

note that this result implies an additional constraint that $\mu>\ln \left[x_{0}\left(\frac{1+\gamma \delta}{1+\delta}\right)^{1 / \delta}\right]$. Then, substituting the corresponding densities and distribution functions into (12) gives 


$$
\begin{aligned}
r & =\gamma \delta x_{0}^{\delta} x_{m}^{-(\delta+1)}\left[1-\left(\frac{x_{m}}{x_{0}}\right)^{-\delta}\right]^{\gamma-1} \Phi\left(\frac{\ln x_{m}-\mu}{\sigma}\right) \\
& \times\left\{\gamma \delta x_{0}^{\delta} x_{m}^{-(\delta+1)}\left[1-\left(\frac{x_{m}}{x_{0}}\right)^{-\delta}\right]^{\gamma-1} \Phi\left(\frac{\ln x_{m}-\mu}{\sigma}\right)\right. \\
& \left.+\frac{1}{\sqrt{2 \pi} x_{m} \sigma} \exp \left(-\frac{1}{2}\left(\frac{\ln x_{m}-\mu}{\sigma}\right)^{2}\right)\left(1-\left[1-\left(\frac{x_{m}}{x_{0}}\right)^{-\delta}\right]^{\gamma}\right)\right\}^{-1}
\end{aligned}
$$

It guarantees that 13 is continuous and smooth. Note that the number of parameters is reduced to four.

The cdf of the composite Lognormal-Stoppa distribution is provided by

$$
F(x)= \begin{cases}r \frac{\Phi\left(\frac{\ln x-\mu}{\sigma}\right)}{\Phi\left(\frac{\ln x_{m}-\mu}{\sigma}\right)}, & 0<x \leq x_{m} \\ r+(1-r) \frac{\left[1-\left(\frac{x}{x_{0}}\right)^{-\delta}\right]^{\gamma}-\left[1-\left(\frac{x_{m}}{x_{0}}\right)^{-\delta}\right]^{\gamma}}{1-\left[1-\left(\frac{x_{m}}{x_{0}}\right)^{-\delta}\right]^{\gamma}}, & x_{m}<x<\infty .\end{cases}
$$

Furthermore, the moment of order $k^{\text {th }}$ of the composite LognormalStoppa distribution exists when $\delta>k$. Its analytical expression is given by

$$
\begin{aligned}
E\left(X^{k}\right) & =r \frac{\Phi\left(\frac{\ln x_{m}-\mu-k \sigma^{2}}{\sigma}\right)}{\Phi\left(\frac{\ln x_{m}-\mu}{\sigma}\right)} e^{k \mu+\frac{k^{2} \sigma^{2}}{2}} \\
& +(1-r) \frac{1}{1-\left[1-\left(\frac{x_{m}}{x_{0}}\right)^{-\delta}\right]^{\gamma}} \gamma x_{0}^{k} B e\left(\left(\frac{x_{m}}{x_{0}}\right)^{-\delta} ; 1-\frac{k}{\delta}, \gamma\right),
\end{aligned}
$$


where $B e(\cdot ; \cdot, \cdot)$ represents the incomplete beta function defined by

$$
B e(x ; a, b)=\int_{0}^{x} z^{a-1}(1-z)^{b-1} d z \quad \text { with } \quad a, b>0 .
$$

In preparation for the resampling scheme in the following numerical applications section, a procedure for generating random variates from the composite Lognormal-Stoppa distribution is presented. As the cdf of the Lognormal and Stoppa distributions can be inverted, the inverse transformation method of simulation can be used for this composite family. If $u$ is a value generated from the uniform distribution $U(0,1)$, then a value generated from $(13)$ is obtained as follows.

- If $u \leq r$ then

$$
x=\exp \left\{\mu+\sigma \cdot \Phi^{-1}\left(\frac{u}{r} \Phi\left(\frac{\ln x_{m}-\mu}{\sigma}\right)\right)\right\} .
$$

- If $u>r$ then

$$
x=x_{0}\left\{1-\left(\frac{u-r}{1-r}\left[1-\left(1-\left(\frac{x_{m}}{x_{0}}\right)^{-\delta}\right)^{\gamma}\right]+\left(1-\left(\frac{x_{m}}{x_{0}}\right)^{-\delta}\right)^{\gamma}\right)^{1 / \gamma}\right\}^{-1 / \delta} .
$$

\subsection{Weibull-Stoppa Model}

The composite Weibull-Stoppa model will be also obtained in terms of the mixture model (9). Its density function is given by

$f(x)= \begin{cases}r \frac{1}{1-\exp \left(-\left(\frac{x_{m}}{\phi}\right)^{\tau}\right)}\left(\frac{\tau}{x}\right)\left(\frac{x}{\phi}\right)^{\tau} \exp \left(-\left(\frac{x}{\phi}\right)^{\tau}\right), & 0<x \leq x_{m} \\ (1-r) \frac{\gamma \delta x_{0}^{\delta} x^{-(\delta+1)}\left[1-\left(\frac{x}{x_{0}}\right)^{-\delta}\right]^{\gamma-1}}{1-\left[1-\left(\frac{x_{m}}{x_{0}}\right)^{-\delta}\right]^{\gamma}}, & x_{m}<x<\infty\end{cases}$

with $\phi>0, \gamma>1, \delta>0,0 \leq r \leq 1$ and $\tau>1$ to define a positive modal value. 
Now, again applying the mode-matching conditions, (10) gives

$$
\phi=\left[x_{0}\left(\frac{1+\gamma \delta}{1+\delta}\right)^{1 / \delta}\right]\left(\frac{\tau}{\tau-1}\right)^{1 / \tau} .
$$

Similarly, substituting the corresponding densities and distribution functions into 12 gives

$$
\begin{aligned}
r & =\gamma \delta x_{0}^{\delta} x_{m}^{-(\delta+1)}\left[1-\left(\frac{x_{m}}{x_{0}}\right)^{-\delta}\right]^{\gamma-1}\left(1-\exp \left(-\left(\frac{x_{m}}{\phi}\right)^{\tau}\right)\right) \\
& \times\left\{\gamma \delta x_{0}^{\delta} x_{m}^{-(\delta+1)}\left[1-\left(\frac{x_{m}}{x_{0}}\right)^{-\delta}\right]^{\gamma-1}\left(1-\exp \left(-\left(\frac{x_{m}}{\phi}\right)^{\tau}\right)\right)\right. \\
& \left.+\left(\frac{\tau}{x_{m}}\right)\left(\frac{x_{m}}{\phi}\right)^{\tau} \exp \left(-\left(\frac{x_{m}}{\phi}\right)^{\tau}\right)\left(1-\left[1-\left(\frac{x_{m}}{x_{0}}\right)^{-\delta}\right]^{\gamma}\right)\right\}^{-1} .
\end{aligned}
$$

The cdf of the composite Weibull-Stoppa distribution is yielded by

$$
F(x)= \begin{cases}r \frac{1-\exp \left(-\left(\frac{x}{\phi}\right)^{\tau}\right)}{1-\exp \left(-\left(\frac{x_{m}}{\phi}\right)^{\tau}\right)}, & 0<x \leq x_{m} \\ r+(1-r) \frac{\left[1-\left(\frac{x}{x_{0}}\right)^{-\delta}\right]^{\gamma}-\left[1-\left(\frac{x_{m}}{x_{0}}\right)^{-\delta}\right]^{\gamma}}{1-\left[1-\left(\frac{x_{m}}{x_{0}}\right)^{-\delta}\right]^{\gamma}}, & x_{m}<x<\infty .\end{cases}
$$

Now, $k^{\text {th }}$ order moment of the composite Weibull-Stoppa distribution exists again if $\delta>k$. Its analytical expression is provided by

$$
\begin{aligned}
E\left(X^{k}\right) & =r \frac{\phi^{k} \Gamma\left(1+\frac{k}{\tau}\right)-\Gamma\left(1+\frac{k}{\tau} ;\left(\frac{x_{m}}{\phi}\right)^{\tau}\right)}{\left(1-\exp \left(-\left(\frac{x_{m}}{\phi}\right)^{\tau}\right)\right)} \\
& +(1-r) \frac{1}{1-\left[1-\left(\frac{x_{m}}{x_{0}}\right)^{-\delta}\right]^{\gamma}} \gamma x_{0}^{k} B e\left(\left(\frac{x_{m}}{x_{0}}\right)^{-\delta} ; 1-\frac{k}{\delta}, \gamma\right),
\end{aligned}
$$


where $\Gamma(\cdot)$ and $\Gamma(\cdot ; \cdot)$ are the complete and incomplete gamma functions defined by

$$
\Gamma(a)=\int_{0}^{\infty} z^{a-1} e^{-z} d z \quad \text { and } \Gamma(a ; x)=\int_{0}^{x} z^{a-1} e^{-z} d z \quad \text { with } \quad a, x>0,
$$

respectively and $B e(\cdot ; \cdot, \cdot)$ is the incomplete beta function.

The procedure for generating random variates from the Weibull-Stoppa distribution is also presented. Similar to the previous section, the inverse transformation method of simulation can be applied as the cdf of the Weibull and Stoppa distributions are invertible. If $u$ is a value generated from the uniform distribution $U(0,1)$, then a value generated from 16 can be obtained as follows.

- If $u \leq r$ then

$$
x=-\phi\left\{\ln \left[1-\frac{u}{r}\left(1-\exp \left[-\left(\frac{\theta}{\phi}\right)^{\tau}\right]\right)\right]\right\}^{1 / \tau} .
$$

- If $u>r$ then

$$
x=x_{0}\left\{1-\left(\frac{u-r}{1-r}\left[1-\left(1-\left(\frac{x_{m}}{x_{0}}\right)^{-\delta}\right)^{\gamma}\right]+\left(1-\left(\frac{x_{m}}{x_{0}}\right)^{-\delta}\right)^{\gamma}\right)^{1 / \gamma}\right\}^{-1 / \delta} .
$$

\section{$5 \quad$ Numerical applications}

In this section, the versatility of the composite Lognormal-Stoppa and WeibullStoppa models, as compared with the composite Pareto and composite Lomax families, is tested using the classic Danish fire insurance dataset. The dataset contains 2,492 fire insurance losses in millions of Danish kroner (DKr) from the years 1980 to 1990 inclusively, adjusted to reflect 1985 values. This dataset may be found in the "SMPracticals" add-on package for R, available from the CRAN website http://cran.r-project.org/.

Parameter estimation for all the models considered in this paper has been completed by the method of maximum likelihood (ML)(which is implemented using the function "mle" / "mle2" in R). The ML estimates for the different composite models, together with their corresponding standard errors, are reported in Table 1 . 


\subsection{Model assessment}

Model assessment is presented from two points of view, theoretical plausibility and practical consideration. For the former point, the theoretical plausibility is justified by means of Kullback-Leibler divergence, suggesting an information-criterion based approach. The following four information criterions are used:

1. Negative log-likelihood (NLL): Calculated by taking the negative of the value of the log-likelihood evaluated at the ML estimates.

2. Akaike information criterion (AIC): Calculated by twice the NLL, evaluated at the ML estimates, plus twice the number of estimated parameters

3. Bayesian information criterion (BIC): Obtained as twice the NLL, evaluated at the ML estimates, plus $k \ln (n)$, where $k$ is the number of estimated parameters and $n$ is the sample size)

4. Consistent Akaike Information Criteria (CAIC): A corrected version of the AIC, proposed by Bozdogan (1987) to overcome the tendency of the AIC overestimating the complexity of the underlying model as it lacks the asymptotic property of consistency. In order to calculate the CAIC, a correction factor based on the sample size is used to compensate for the overestimating nature of AIC. The CAIC is defined as twice the NLL plus $k(1+\ln (n))$, again $k$ is the number of free parameters and $n$ refers to the sample size.

Note that for all the information criterion above, smaller values indicate a better fit of the model to the data. The results are shown in Table 1, It can be seen that within each of the Lognormal-composite family and the Weibull-composite family, the model associated with the Stoppa distribution outperforms the ones assocciated with the Pareto or Lomax distributions in all of the goodness-of-fit measures mentioned before. Overall, the WeibullStoppa composite model provides the best fit, again consistently across the different measures, to the data. Illustration of the fit of all the composite models is given in Figure 2 and Figure 3. Upon the recommendation of an anonymous reviewer, we have calculated the values of the NLL for the Lognormall-Stoppa and Weibull-Stoppa distributions under the traditional continuity-differentiability conditions. Those figures are 3858.51 for 
the former model and 3818.79 for the latter one; it is important to mention that the ML estimates obtained by using this approach are located in the neighbourhood of the ones computed under the mode-matching procedure. Probably the reason for that is that local maxima were found for these models and the probabilistic families derived under the mode--matching procedure can be considered a particular case of those ones obtained by using the continuity-differentiability approach. As a side note, an evaluation of the fitted mode is given. The sample mode of the data is 0.8873114 . The Lognormal-Stoppa distribution gives a theoretical mode of 1.06041 and a mixing weight of $r=0.1657338$; the Lognormal-Lomax distribution gives a mode of 1.072794 and a weight of $r=0.2381526$ and the Lognormal-Pareto distribution gives 1.103568 and 0.2900379 respectively. For the composite models based on the Weibull distribution, we have a mode of 0.9454775 and a weight of $r=0.08148809$ for the Weibull-Stoppa distribution, a mode of 0.9647542 and a weight of $r=0.1063022$ for the Weibull-Lomax distribution and a mode of 0.9913752 and a weight of $r=0.1394686$ for the WeibullPareto distribution. The Lognormal-Stoppa distribution and the WeibullStoppa distribution have the closest fitted mode to the sample mode within their own family of distributions; and considering the two families together, Weibull-Stoppa has the closest fitted mode.

For the latter point, applications of the composite model, especially in the context of actuarial studies, involve mostly calculations done using the distribution function of the fitted model, for instance, the expected loss above a threshold and the value-at-risk. Hence, it is useful to express the fit of the model to the data in terms of distribution functions. In particular, it is suggested to use the following three empirical distribution function (EDF) goodness-of-fit measures to quantify the "distance" between the empirical distribution function constructed from the data and the cumulative distribution function of the fitted models. They are the Kolmogorov-Smirnov test statistics, the Cramer-von Mises test statistics and the Anderson-Darling test statistics (Rizzo, 2009). The definition of the test statistics are given as follows: Denote the cumulative distribution function of the fitted model by $\hat{F}$, the original data by $x_{1}, \ldots, x_{N}$ and the ordered data in increasing magnitude by $x_{(1)}, \ldots, x_{(N)}$, then we have:

1. Kolmogorov-Smirnov $(\mathrm{KS})$ test statistics: $D=\max \left(D^{+}, D^{-}\right)$, where

$$
\left.D^{+}=\max _{1 \leq j \leq N}\left\{\frac{j}{N}-\hat{F}\left(x_{(j)}\right)\right)\right\}, D^{-}=\max _{1 \leq j \leq N}\left\{\hat{F}\left(x_{(j)}\right)-\frac{j-1}{N}\right\} .
$$




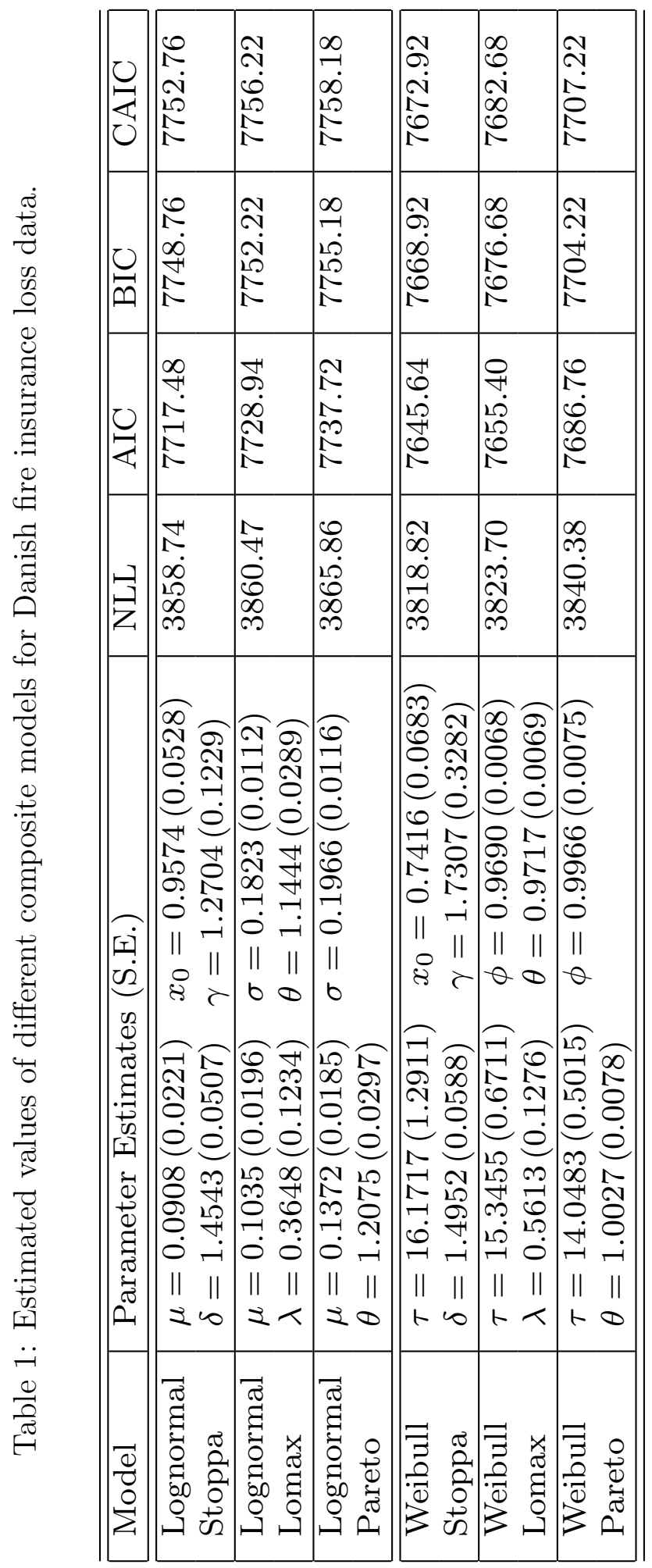


Fitted Density Curves for the Composite Lognormal Models

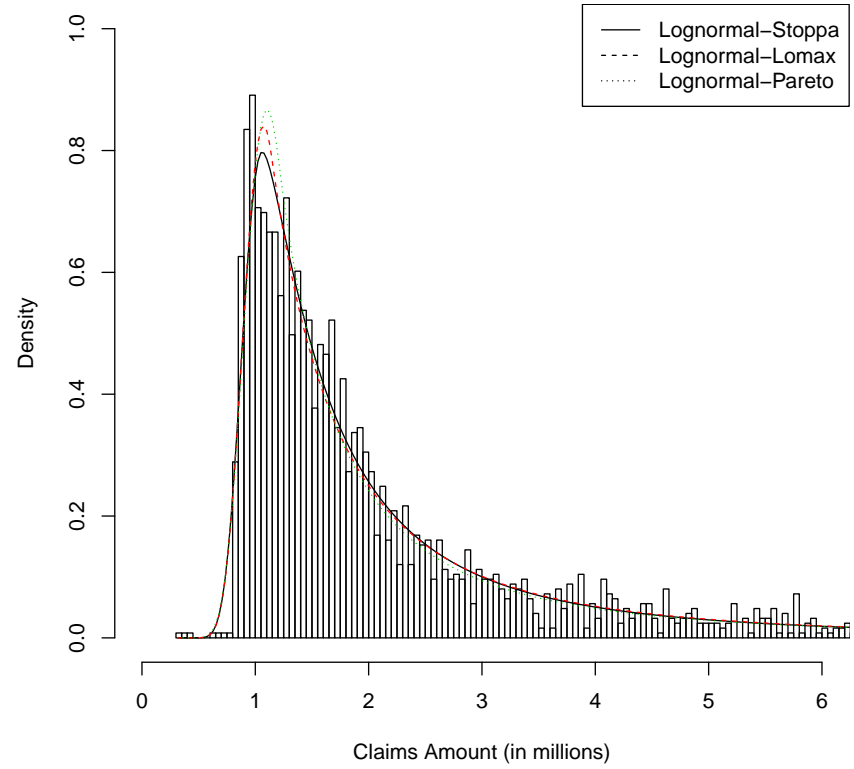

Fitted Density Curves for the Composite Lognormal Models

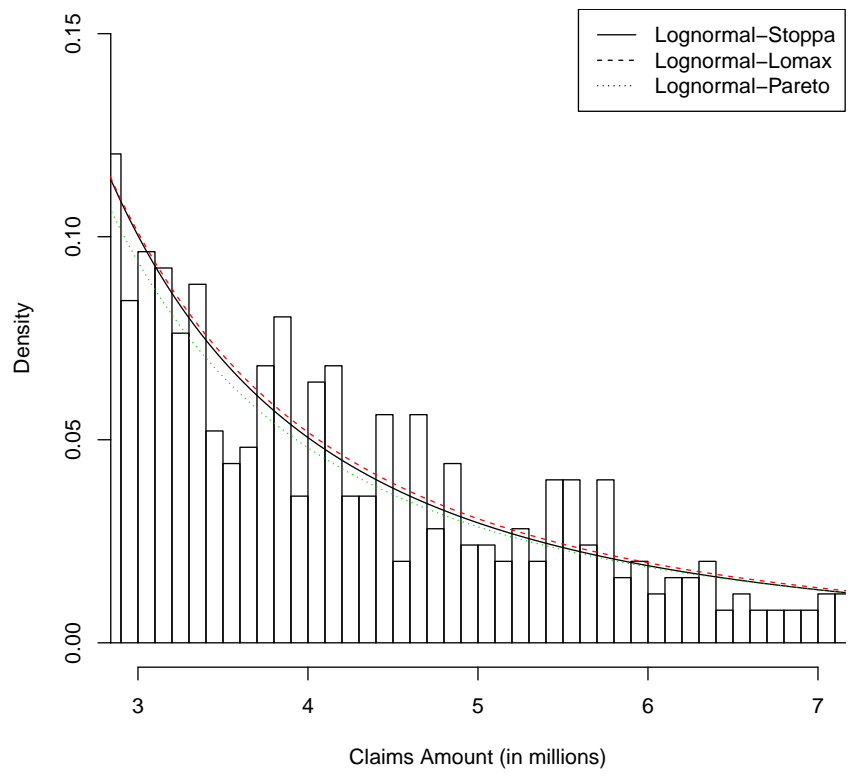

Figure 2: Comparison of empirical histogram for the Danish fire insurance data, Lognormal-Stoppa (solid), Lognormal-Lomax (dashed) and Lognormal-Pareto (dotted). 

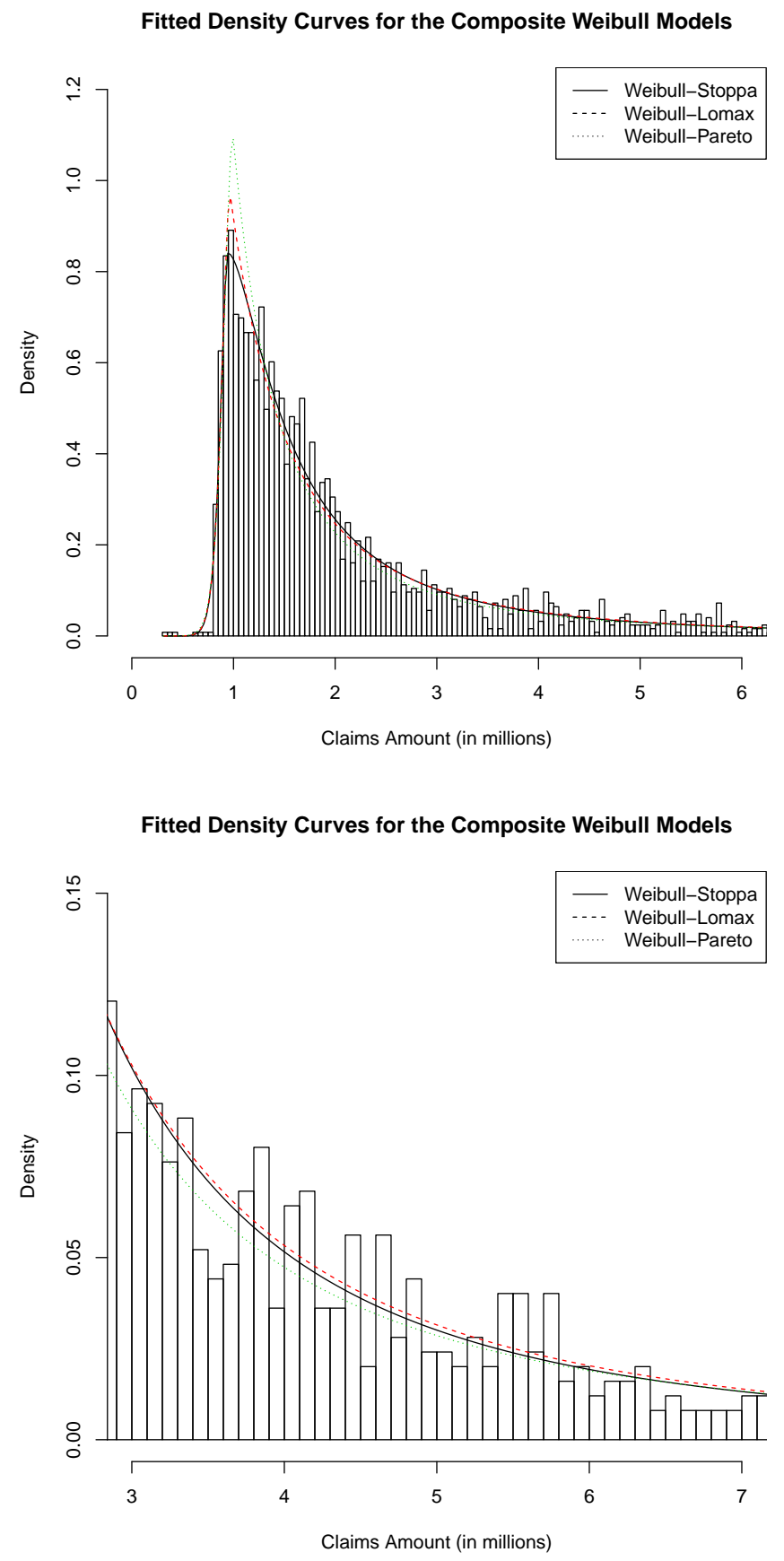

Figure 3: Comparison of empirical histogram for the Danish fire insurance data, Weibull-Stoppa (solid), Weibull-Lomax (dashed) and Weibull-Pareto (dotted). 
2. Cramer-von Mises (CvM) test statistics:

$$
W^{2}=\sum_{j=1}^{N}\left[\hat{F}\left(x_{(j)}\right)-\frac{2 j-1}{2 N}\right]^{2}+\frac{1}{12 N} .
$$

3. Anderson-Darling (AD) test statistics:

$$
A^{2}=-N-\frac{1}{N} \sum_{j=1}^{N}\left[(2 j-1) \log \left(\hat{F}\left(x_{(j)}\right)\right)+(2 n+1-2 j) \log \left(1-\hat{F}\left(x_{(j)}\right)\right)\right] .
$$

For all the EDF goodness-of-fit measures above, smaller values indicate a better fit of the model to the data. The results are summarized in Table 2. It can be seen that using the $\mathrm{CvM}$ and $\mathrm{AD}$ measure, within each of the Lognormal-composite family and the Weibull-composite family, the composite model associated with the Stoppa distribution gives the best fit. In fact, unlike the case in the information-criterion section, the Lognormal-Stoppa and Weibull-Stoppa models are the best two models considering all the models at once. In the KS case, it is observed that while the Weibull-Stoppa model is still the best overall, the Lognormal-Lomax model outperforms the Lognormal-Stoppa model by a slight margin. The reason is believed to be that Kolmogorov-Smirnov test is relatively insensitive to deviations occuring in the tail (Mason and Schuenemeyer, 1983). Besides, since the improvement brought by using the Stoppa distribution in place of the Lomax distribution only occurs in the tail, the KS test statistics may not have fully reflected the improvement, resulting in the slightly superior fit of the Lognormal-Lomax model over the Lognormal-Stoppa model.

Note that the test statistics not only provide a way to measure the fit in terms of distribution functions, but also allow us to perform hypothesis testing for model validation purposes. We remark that to perform the goodnessof-fit tests, it is required the proposed model is specified completely, i.e. parameters need to be specified too. In the case where parameters are estimated from data, the critical values produced using the standard procedure are no longer valid (Babu and Rao, 2004). To circumvent this problem, we use the bootstrap method. The validity is justified by the work of Babu and Rao (2004), in which the consistency of the bootstrap method estimating the null distribution of the goodness-of-fit test statistics was shown. We present a brief outline of the bootstrap procedure. Denote the data by $x_{1}, \ldots, x_{N}$. For each proposed composite model, fit the model to the data. Then, 
Table 2: EDF goodness-of-fit measures of the composite models.

\begin{tabular}{lrrr}
\hline \hline & \multicolumn{3}{c}{ test statistics } \\
\cline { 2 - 4 } Model & KS & CvM & AD \\
\hline Lognormal-Pareto & 0.032304 & 0.47814 & 3.15964 \\
Lognormal-Lomax & 0.019515 & 0.21406 & 1.95087 \\
Lognormal-Stoppa & 0.019739 & 0.14493 & 1.70092 \\
Weibull-Pareto & 0.051729 & 1.51904 & 7.33822 \\
Weibull-Lomax & 0.025506 & 0.33780 & 1.90971 \\
Weibull-Stoppa & 0.017340 & 0.12615 & 0.88225 \\
\hline \hline
\end{tabular}

1. Compute the goodness-of-fit test statistics, $t_{K S}, t_{C v M}, t_{A D}$.

2. Use the fitted model to perform parametric bootstrapping.

- Generate $\mathrm{M}$ sets of resampled data, denote as $\hat{x}_{1}^{(i)}, \ldots, \hat{x}_{N}^{(i)}, i=$ $1, \ldots, M$

- For each set of the resampled data, fit the composite model and compute the test statistics, $t_{K S}^{(i)}, t_{C v M}^{(i)}, t_{A S}^{(i)}, i=1, \ldots, M$.

3. The $p$-value of the respective original test statistics are given by

$$
\frac{\#\left\{i: t_{K S}^{(i)} \geq t_{K S}\right\}}{M}, \frac{\#\left\{i: t_{C v M}^{(i)} \geq t_{C v M}\right\}}{M}, \frac{\#\left\{i: t_{A D}^{(i)} \geq t_{A D}\right\}}{M} .
$$

The $p$-value of the test statistics, computed using $M=10000$ simulations, are presented in Table 3. We remark that while an extremely small $p$-value may lead to a confident rejection of the null hypothesis that the data comes from the proposed model, and in general a larger $p$-value is favourable, a $p$-value being large doesn't serve well as evidence of the model being correct especially when there are other models with a $p$-value of comparable magnitude. It can be seen that none of the models are rejected, validating that the models are statistically legitimate candidates. In addition, the composite-Stoppa models, together with the composite-Lomax models in this case, have relatively high (hence favourable) $p$-values across different EDF goodness-of-fit measures. 
Table 3: $p$-values of the EDF goodness-of-fit measures of the composite models, computed with 10000 sets of bootstrap resamples.

\begin{tabular}{lrrr}
\hline \hline & \multicolumn{3}{c}{$p$-value of the test statistics } \\
\cline { 2 - 4 } Model & KS & CvM & AD \\
\hline Lognormal-Pareto & 0.5314 & 0.5527 & 0.6968 \\
Lognormal-Lomax & 0.9741 & 0.9219 & 0.9410 \\
Lognormal-Stoppa & 0.9360 & 0.9530 & 0.9440 \\
Weibull-Pareto & 0.5071 & 0.5086 & 0.5324 \\
Weibull-Lomax & 0.7949 & 0.7760 & 0.8364 \\
Weibull-Stoppa & 0.6955 & 0.7104 & 0.7571 \\
\hline \hline
\end{tabular}

\subsection{Applications}

In this section, investigation on two practical concerns, namely the high quantiles and the probable maximal loss, is presented. As both the quantities are related to the distribution function of the model, the performance in these terms is expected to be in line with the results shown in the EDF goodnessof-fit section.

\subsubsection{Estimation of high quantiles}

It is often convenient for practitioners to obtain reliable information about the tail of the claim size distribution. A measure that yields an acceptable knowledge of the right tail of the model is the high quantiles. Empirical and fitted quantiles in the extreme portion of the tail for composite Lognormal and composite Weibull are given in Table 4 and Table 5 respectively. The empirical quantiles have been computed using the Type 8 quantile algorithm suggested by Hyndman and Fan (1996). It is our interest to analyze how much theoretical tail quantiles of each fitted composite model deviate from the empirical quantiles in the extreme portion of the tail. It can be seen that the composite Stoppa models demonstrate a better fit to the data in the high quantiles, likewise suggesting it being a favourable model for the given data. The Lognormal-Pareto and Weibull-Pareto distributions tend to overestimate the exteme tail quantiles whereas Lognormal-Lomax and Weibull-Lomax composite models underestimate them. We remark that in- 
terpretation of the results obtained from this table needs to be prudently made given that the extreme-value data is scarce. To illustrate the point, note that the sample size of the dataset is only 2492 while the value of the 99.99\% empirical quantile represents an event that occurs 1 in 10000 times.

Table 4: Empirical and fitted composite Lognormal model quantiles.

\begin{tabular}{lrrrr}
\hline \hline & & \multicolumn{3}{c}{ Composite Lognormal } \\
\cline { 3 - 5 } Quantiles & Empirical & Pareto & Lomax & Stoppa \\
\hline 0.50 & 1.634 & 1.572 & 1.611 & 1.590 \\
0.90 & 5.086 & 5.282 & 5.164 & 5.028 \\
0.95 & 8.459 & 8.902 & 8.249 & 8.134 \\
0.99 & 24.870 & 29.903 & 23.750 & 24.683 \\
0.999 & 146.010 & 169.227 & 104.835 & 120.322 \\
0.9995 & 199.020 & 285.259 & 163.540 & 193.801 \\
0.9999 & 263.250 & 958.261 & 458.572 & 586.128 \\
\hline \hline
\end{tabular}

Table 5: Empirical and fitted composite Weibull model quantiles.

\begin{tabular}{lrrrr}
\hline \hline & & \multicolumn{3}{c}{ Composite Weibull } \\
\cline { 3 - 5 } Quantiles & Empirical & Pareto & Lomax & Stoppa \\
\hline 0.50 & 1.634 & 1.542 & 1.615 & 1.632 \\
0.90 & 5.086 & 5.522 & 5.201 & 5.129 \\
0.95 & 8.459 & 9.566 & 8.203 & 8.211 \\
0.99 & 24.870 & 34.262 & 22.648 & 24.223 \\
0.999 & 146.010 & 212.586 & 92.931 & 113.126 \\
0.9995 & 199.020 & 368.271 & 141.649 & 179.885 \\
0.9999 & 263.250 & 1319.032 & 376.050 & 527.741 \\
\hline \hline
\end{tabular}

\subsubsection{Probable maximum loss}

We conclude this section by presenting the probable maximum loss (PML) for the family of composite models described in this paper. In general terms, 
the PML can be defined as the worst loss likely to happen (Cebrián et al (2003)). To be specific, given a random variable $N$ that follows a Poisson distribution with mean $\zeta$, and $X_{1}, \ldots, X_{N}$ a sequence of independent and identically distributed random variables, define a sequence of maxima $M_{N}=$ $\max \left(X_{1}, \ldots, X_{N}\right)$ with cdf $F_{M_{N}}$. Then, the PML is the quantile function of the maximum loss $M_{N}$ and is given by $P M L=F_{M_{N}}^{-1}(q)$. For implementation, the value of $\zeta$ is needed, we follow Pigeon and Denuit (2011) and use the average annual frequency as an estimate, which is given by $\hat{\zeta}=226.5455$. Results of PML for values of $q=0.90, q=0.95$ and $q=0.99$ are shown in Table 6.

Table 6: Probable maximal losses for different values of $q$.

\begin{tabular}{lrrr}
\hline \hline \multirow{2}{*}{ Model } & \multicolumn{3}{c}{ Probable Maximal Loss $q$} \\
\cline { 2 - 4 } & 0.90 & 0.95 & 0.99 \\
\hline Lognormal-Pareto & 301.20 & 517.86 & 1766.67 \\
Lognormal-Lomax & 171.10 & 271.32 & 770.04 \\
Lognormal-Stoppa & 229.74 & 376.89 & 1156.00 \\
Weibull-Pareto & 390.10 & 690.25 & 2513.00 \\
Weibull-Lomax & 147.65 & 228.48 & 613.17 \\
Weibull-Stoppa & 195.11 & 315.78 & 939.37 \\
\hline \hline
\end{tabular}

\section{Conclusions}

A new composite family for modeling claims data of mixed sizes has been proposed and its performance is compared with existing models using a classic insurance dataset. The composite model is developed using a mode-matching procedure. The Lognormal or Weibull distribution is used up to its mode as the first component of the spliced model and thereafter, the Stoppa distribution is used as the second component. The Goodness-of-fit has been analyzed using two different methodologies, an information-criterion approach and an empirical-distribution-function approach. The choices are made to address theoretical plausibility and practical considerations. Numerical results show that the composite Stoppa model outperforms the existing composite models under six goodness-of-fit measures in the context of the Danish fire insurance 
data set. This together with its simple implementation make it an appealing tool to model claims of mixed sizes. Finally, although the simplification and numerical implemetation of the resulting models could be cumbersome and tedious, it might be interesting to consider a further extension of the composite Stoppa models under the traditional continuity-differentiability approach.

\section{References}

Babu, G.J. and Rao, C.R. (2004). Goodness-of-fit tests when parameters are estimated Sankhya: The Indian Journal of Statistics, 66, 1, 63-74.

Bozdogan, H. (1987). Model selection and Akaike's Information Criterion (AIC): The general theory and its analytical extensions. Psychometrika, 52, 3, 345-370.

Burkhauser, R.V., Feng, S. and Larrimore, J. (2010). Improving imputations of top incomes in the public-use current population survey by using both cell-means and variances. Economics Letters, 108, 1, 69-72.

Cebrián, A., Denuit, M. and Lambert, Ph. (2003). Generalized Pareto fit to the society of actuaries' large claims data base. North American Actuarial Journal, 7, 3, 18-36.

Ciumara, R. (2006). An actuarial model based on the composite WeibullPareto distribution. Mathematical Report-Bucharest, 8(4), 401-414.

Cooray, K. and Ananda, M.M.A. (2005). Modeling actuarial data with a compostie Lognormal-Pareto model. Scandinavian Actuarial Journal, $5,321-334$.

Hyndman, R.J. and Fan, Y. (1996). Sample quantiles in statistical packages. American Statistician, 50, 361-365.

Kleiber, C. and Kotz, S. ( 2003 ). Statistical Size Distributions in Economics and Actuarial Sciences. Hoboken, NJ: John Wiley \& Sons.

Klugman, S.A., Panjer, H.H. and Willmot, G.E. (2008). Loss Models: From Data to Decisions. Third Edition. Wiley. 
Mason, D. M. and Schuenemeyer, J. H. (1983). A modified KolmogorovSmirnov test sensitive to tail alternatives. Ann. Statist., 11, 933-946.

Nadarajah, S. and Bakar, S.A.A. (2014). New composite models for the Danish fire insurance data. Scandinavian Actuarial Journal, 2, 180187.

Pigeon, M and Denuit, M. (2011). Composite Lognormal-Pareto model with random threshold. Scandinavian Actuarial Journal, 3, 177-192.

Rizzo, M.L. (2009). New goodness-of-fit tests for Pareto distributions. Astin Bulletin, 39, 2, 691-715.

Scollnik, D. P. M. (2007). On composite Lognormal-Pareto models. Scandinavian Actuarial Journal, 1, 20-33.

Scollnik, D. P. M. and Sun, C. (2012). Modeling with Weibull-Pareto models. North American Actuarial Journal, 16, 2, 260-272.

Stoppa, G. (1990). Proprietà campionarie di un nuovo modello Pareto generalizzato. Atti XXXV Riunione Scientifica della Società Italiana di Statistica, Padova: Cedam, 137-144. 


\section{University Library}

\section{- M M I N E R VA A gateway to Melbourne's research publications}

Minerva Access is the Institutional Repository of The University of Melbourne

Author/s:

Calderín-Ojeda, E;Kwok, CF

Title:

Modeling claims data with composite Stoppa models

Date:

2016

Citation:

Calderín-Ojeda, E. \& Kwok, C. F. (2016). Modeling claims data with composite Stoppa models. Scandinavian Actuarial Journal, 9 (9), pp.817-836. https:// doi.org/10.1080/03461238.2015.1034763.

Persistent Link:

http://hdl.handle.net/11343/120649 